\title{
Electron impact excitation collision strength for transitions in $\mathrm{C} \mathrm{II}{ }^{\star}$
}

\author{
S. S. Tayal
}

\author{
Department of Physics, Clark Atlanta University, Atlanta, GA 30314, USA \\ e-mail: stayal@cau.edu
}

Received 25 April 2008 / Accepted 20 May 2008

\begin{abstract}
Aims. Oscillator strengths and electron impact excitation collision strengths for C II lines among the fine-structure levels are calculated. Thermally averaged collision strengths are presented as a function of electron temperature for application to astrophysical plasmas.

Methods. An accurate description of the target wave functions has been obtained in the multiconfiguration Hartree-Fock approach with flexible non-orthogonal orbitals. The 42 fine-structure levels of the $2 \mathrm{~s}^{2} 2 \mathrm{p}, 2 \mathrm{~s} 2 \mathrm{p}^{2}, 2 \mathrm{~s}^{2} 3 l(l=0-2), 2 \mathrm{p}^{3}, 2 \mathrm{~s}^{2} 4 l(l=0-3), 2 \mathrm{~s} 2 \mathrm{p} 3 \mathrm{~s}$ and $2 s^{2} 5 l(l=0-2)$ configurations have been included in the scattering calculation. A second calculation with lowest 35 levels in the close-coupling expansion has also been carried out to check channel coupling effects on collision strengths. The continuum functions have been represented by the use of B-spline basis. The B-spline R-matrix method in the framework of Breit-Pauli approximation has been used to investigate the electron impact excitation of forbidden and allowed transitions in C II.

Results. The present cross sections for the resonance ${ }^{2} \mathrm{P}^{\circ}-2 \mathrm{~s} 2 \mathrm{p}^{2}{ }^{2} \mathrm{D},{ }^{2} \mathrm{~S}$ and intercombination ${ }^{2} \mathrm{P}^{\circ}-2 \mathrm{~s} 2 \mathrm{p}^{2}{ }^{4} \mathrm{P}$ transitions are in very good agreement with the electron energy-loss merged-beams experiment. Oscillator strengths and transition probabilities for $\mathrm{C}$ II lines normally compare very well with previous calculation. The effective collision strengths are obtained by integrating total resonant and non-resonant collision strengths over a Maxwellian distribution of electron energies and these are presented over a wide temperature range suitable for modeling of astrophysical plasmas. Our effective collision strengths show significant differences with those from previous calculations for transitions involving higher excitation levels.
\end{abstract}

Key words. atomic data - atomic processes

\section{Introduction}

Studies of temperatures, densities and turbulence and flow velocities of the outer atmospheres of individual stars and their variation with global stellar properties such as effective temperature, surface gravity and dust content contribute to the understanding of evolution of stars. A large amount of atomic data are needed to compute the energy transport through the outer layers of a star. The intercombination lines due to $2 \mathrm{~s}^{2} 2 \mathrm{p}^{2} \mathrm{P}_{J^{-}}^{\circ}$ $2 \mathrm{~s} 2 \mathrm{p}^{2}{ }^{4} \mathrm{P}_{J^{\prime}}$ transitions in $\mathrm{C}$ II can provide excellent density diagnostics in the range $10^{9} \leq N_{\mathrm{e}} \geq 10^{12} \mathrm{~cm}^{-3}$. The C II lines due to the $2 \mathrm{~s}^{2} 2 \mathrm{p}{ }^{2} \mathrm{P}_{J}^{\circ}-2 \mathrm{~s} 2 \mathrm{p}^{2}{ }^{2} \mathrm{D}_{J^{\prime}}$ and $2 \mathrm{~s} 2 \mathrm{p}^{2}{ }^{4} \mathrm{P}_{J}-2 \mathrm{p}^{3}{ }^{4} \mathrm{~S}_{J^{\prime}}^{\circ}$ transitions are also electron density sensitive. The $\mathrm{C}$ II intercombination lines $2 \mathrm{~s}^{2} 2 \mathrm{p}{ }^{2} \mathrm{P}_{J}^{\circ}-2 \mathrm{~s} 2 \mathrm{p}^{2}{ }^{4} \mathrm{P}_{J^{\prime}}$ are useful to investigate turbulence and electron density in non-coronal stars. These lines are also important for the abundance determinations in both galactic and extragalactic stellar atmospheres and diffuse interstellar gas. Interstellar densities can be derived for $\mathrm{H}$ II regions by using C II $1037 \AA$ line due to the $2 \mathrm{~s}^{2} 2 \mathrm{p}^{2} \mathrm{P}_{3 / 2}^{\circ}-2 \mathrm{~s} 2 \mathrm{p}^{2}{ }^{2} \mathrm{~S}_{1 / 2}$ transition. The C II $\lambda \lambda 1334.53,1335.71$ lines due to the $2 \mathrm{~s}^{2} 2 \mathrm{p}{ }^{2} \mathrm{P}_{J}^{\circ}-2 \mathrm{~s} 2 \mathrm{p}^{2}{ }^{2} \mathrm{D}_{J^{\prime}}$ transitions are strong interstellar features in the STIS/HST spectra of Carina nebula (Walborn et al. 2002).

The oscillator strengths and scattering of electrons from C II have been studied by several theoretical and experimental groups in the past. The theoretical studies of oscillator strengths include configuration-interaction (CI) calculations of Correge \& Hibbert (2002) and Lennon et al. (1985) using the atomic structure

* Tables 4 and 5 are only available in electronic form at the CDS via anonymous ftp to cdsarc.u-strasbg.fr (130.79.128.5) or via http://cdsweb.u-strasbg.fr/cgi-bin/qcat?J/A+A/486/629 code CIV3 (Hibbert 1975); Nussbaumer \& Storey (1981) and Galavis et al. (1998) using computer code SUPERSTRUCTURE (Eissner \& Nussbaumer 1974), and multiconfiguration HartreeFock (MCHF) calculations of Brage et al. (1995) and Tachiev \& Froese Fischer (2000). Previous theoretical calculations for electron impact excitation collision strengths include close-coupling calculations of Lennon et al. (1985); Hayes \& Nussbaumer (1984) and R-matrix calculations of Keenan et al. (1986); Luo \& Pradhan (1990); Blum \& Pradhan (1991, 1992); Wilson \& Bell (2002) and Wilson et al. (2005). Blum \& Pradhan (1992) and Wilson et al. (2005) included 10 and 16 LS target states respectively in the close-coupling expansion in their R-matrix calculations in LS coupling and then used an algebraic transformation to obtain collision strengths for fine-structure transitions. Measured and calculated excitation cross sections for the $2 \mathrm{~s}^{2} 2 \mathrm{p}^{2} \mathrm{P}^{\circ}-2 \mathrm{~s} 2 \mathrm{p}^{2}{ }^{4} \mathrm{P},{ }^{2} \mathrm{D}$ and ${ }^{2} \mathrm{~S}$ transitions have been reported by Smith et al. (1996). The measurements of absolute cross sections were carried out using the energy-loss merged-beams method. The LS coupling R-matrix calculations were used to compare with the experiment as the fine-structure components were not resolved. A good agreement between theory and experiment was noted. Measured cross sections for the electron impact excitation of the $2 \mathrm{~s}^{2} 2 \mathrm{p}{ }^{2} \mathrm{P}^{\circ}-2 \mathrm{~s} 2 \mathrm{p}^{2}{ }^{2} \mathrm{D}$ transition in C II were reported by Lafyatis \& Kohl (1987) and Williams et al. (1998).

This paper represents another attempt to improve the theoretical aspect of electron impact excitation of C II by improving the description of target wave functions and by properly including resonances in the collision strengths. Higher degree of sophistication is required to accurately calculate the position of resonances, and a fine energy mesh is needed to resolve 
resonances. We used non-orthogonal orbitals that are optimized on separate states to account for the term dependence of target wave functions. We used B-spline basis for the description of continuum functions and did not impose any orthogonality constraint between continuum functions and the valence spectroscopic and correlated atomic orbitals (Zatsarinny \& Tayal 2001; Zatsarinny \& Bartschat 2004; Zatsarinny 2006). This allowed us to avoid potential inconsistencies between the continuum and bound parts of the close-coupling expansion. We included 42 fine-structure levels arising from the lowest 23 LS target terms of the $2 s^{2} 2 p, 2 s 2 p^{2}, 2 s^{2} 3 s, 2 s^{2} 3 p, 2 p^{3}, 2 s^{2} 3 d, 2 s^{2} 4 s$, $2 \mathrm{~s}^{2} 4 \mathrm{p}, 2 \mathrm{~s} 2 \mathrm{p} 3 \mathrm{~s}, 2 \mathrm{~s}^{2} 4 \mathrm{~d}, 2 \mathrm{~s}^{2} 4 \mathrm{f}, 2 \mathrm{~s}^{2} 5 \mathrm{~s}, 2 \mathrm{~s}^{2} 5 \mathrm{p}, 2 \mathrm{~s}^{2} 5 \mathrm{~d}, 2 \mathrm{~s}^{2} 5 \mathrm{f}$ and $2 \mathrm{~s}^{2} 6 \mathrm{~s}$ configurations in the close-coupling expansion. The inclusion of these levels in the expansion ensures channel coupling effects reasonably well up to the excited $2 \mathrm{~s}^{2} 5 \mathrm{~d}{ }^{2} \mathrm{D}_{3 / 2,5 / 2}$ levels around 1.7 Ryd. The higher excited levels belonging to the $2 \mathrm{~s}^{2} 5 \mathrm{f}^{2} \mathrm{~F}^{\circ}$, $2 s^{2} 6 s{ }^{2} \mathrm{~S}$ and $2 \mathrm{~s} 2 \mathrm{p} 3 \mathrm{p}{ }^{2} \mathrm{P}$ terms may have significant coupling with higher excited levels that lie above these levels. Our results agree very well with the available measured absolute direct excitation cross sections (Smith et al. 1996). In the present paper we present electron impact collision strengths and rates for fine-structure transitions among the $2 \mathrm{~s}^{2} 2 \mathrm{p}^{2} \mathrm{P}_{1 / 2,3 / 2}^{\circ}, 2 \mathrm{~s} 2 \mathrm{p}^{2}$ ${ }^{4} \mathrm{P}_{5 / 2,3 / 2,1 / 2}, 2 \mathrm{~s} 2 \mathrm{p}^{2}{ }^{2} \mathrm{D}_{5 / 2,3 / 2}, 2 \mathrm{~s} 2 \mathrm{p}^{2}{ }^{2} \mathrm{P}_{1 / 2,3 / 2}, 2 \mathrm{~s} 2 \mathrm{p}^{2}{ }^{2} \mathrm{~S}_{1 / 2}, 2 \mathrm{~s}^{2} 3 l$ $(l=0-2){ }^{2} \mathrm{~S}_{1 / 2},{ }^{2} \mathrm{P}_{1 / 2,3 / 2}^{\circ},{ }^{2} \mathrm{D}_{3 / 2,5 / 2}, 2 \mathrm{p}^{3}{ }^{4} \mathrm{~S}_{3 / 2}^{\circ},{ }^{\circ} \mathrm{D}_{3 / 2,5 / 2}^{\circ},{ }^{2} \mathrm{P}_{1 / 2,3 / 2}^{\circ}$, $2 \mathrm{~s}^{2} 4 l(l=0-3){ }^{2} \mathrm{~S}_{1 / 2},{ }^{2} \mathrm{P}_{1 / 2,3 / 2}^{\circ},{ }^{2} \mathrm{D}_{3 / 2,5 / 2},{ }^{2} \mathrm{~F}_{5 / 2,7 / 2}^{\circ}, 2 \mathrm{~s}^{2} 5 l(l=0-2)$ ${ }^{2} \mathrm{~S}_{1 / 2},{ }^{2} \mathrm{P}^{\circ} 1 / 2,3 / 2,{ }^{2} \mathrm{D}_{3 / 2,5 / 2}$ and $2 \mathrm{~s} 2 \mathrm{p} 3 \mathrm{~s}{ }^{2} \mathrm{P}_{1 / 2,3 / 2}^{\circ}$ levels over a temperature range that is suitable for astrophysical plasma modeling calculations. We also present oscillator strengths for allowed transitions among the lowest 26 fine-structure levels and some other transitions from these levels to the higher excitation levels.

\section{Computational methods}

We used non-orthogonal orbitals to represent different C II states. The non-orthogonal orbitals provide much greater flexibility in the choice of wave functions than the orthogonal orbitals and also allow to include correlation with a reasonable number of configurations and correlated orbitals. Our calculations are performed using the multiconfiguration Hartree-Fock (MCHF) method (Froese Fischer 1991; Zatsarinny \& Froese Fischer 2000). In the MCHF approach each atomic state is represented by an atomic state function

$\Psi(\alpha L S)=\sum_{i} c_{i} \Phi\left(\alpha_{i} L S\right)$

where the configuration state functions $\Phi\left(\alpha_{i} L S\right)$ are constructed from one-electron functions and $\alpha_{i}$ defines the coupling of angular momenta of the electrons. The $J$-dependent atomic state functions are written as a sum over different LS values which couple to give the total angular momentum $J$

$\Psi(\alpha J)=\sum_{j} a_{j} \Psi\left(\alpha_{j} L_{j} S_{j} J\right)$.

The C II wave functions exhibit large correlation corrections and significant term dependence of the one-electron orbitals. The non-orthogonal orbitals are optimized for each atomic state separately. We began with the Hartee-Fock (HF) calculation for the $1 s, 2 s$ and $2 p$ orbitals for the ground $2 s^{2} 2 p$ configuration terms and then determined separate sets of excited orbitals for various symmetries of even and odd parities. A set of correlation s, p, $\mathrm{d}$ and $\mathrm{f}$ orbitals was determined by optimization on the ground state and another set of s, p, d and f correlation orbitals was optimized on the $2 \mathrm{~s} 2 \mathrm{p}^{2}{ }^{2} \mathrm{D}$ state to account for the interaction between the $2 s^{2} n$ d Rydberg series and $2 \mathrm{~s} 2 \mathrm{p}^{2}$ perturber states. The mean radii of the correlation orbitals are comparable to the spectroscopic orbitals and thus the correlation corrections are very well represented. The correlation corrections for C II are significant. The spectroscopic and correlation functions are used to construct CI expansions for different atomic states by allowing one-electron and two-electron excitations from all the basic configurations $2 \mathrm{~s}^{2} 2 \mathrm{p}, 2 \mathrm{~s} 2 \mathrm{p}^{2}, 2 \mathrm{~s} 2 \mathrm{p} 3 \mathrm{~s}, 2 \mathrm{~s}^{2} n l(n=3-5 ; l=0-2)$ and $2 \mathrm{~s} 2 \mathrm{p} 3 \mathrm{p}$ used in our calculation. We used 40 non-orthogonal oneelectron orbitals to construct CI expansions for levels of even parity and odd parity. In the construction of CI expansions for fine-structure levels with various $J$ and $\pi$ we used configurations generated in this excitation scheme for the atomic LS states and with insignificant configurations with coefficients less than 0.003 omitted from the expansions. We included 183 and 194 configurations in the CI expansions of levels of odd parity and even parity respectively in our final calculation. Our calculation takes an account of the important correlation and relaxation effects and of the term dependence of the valence orbitals. These wave functions are then used to calculate the length $\left(f_{L}\right)$ and velocity $\left(f_{V}\right)$ forms of oscillator strengths and transition probabilities for transitions among the fine-structure levels.

The theoretical approach and codes used in the calculation of collision strengths have been described by Zatsarinny (2006), and here we present a brief outline. The wave function describing the total $\mathrm{e}+\mathrm{C}$ II system in the internal region is expanded in terms of energy-independent functions

$\Psi_{k}=A \sum_{i j} a_{i j k} \overline{\Phi_{i}} u_{j}(r)$,

where $\overline{\Phi_{i}}$ are channel functions formed from the multiconfigurational functions of the 42 target levels and $u_{j}$ are the radial basis functions describing the motion of the scattering electron. The operator A antisymmetrizes the wave function and expansion coefficients $a_{i j k}$ are determined by diagonalizing the $(N+1)$-electron Hamiltonian. In our calculation, the radial functions $u_{j}$ are expanded in the B-spline basis as

$u_{j}(r)=\sum_{i} \overline{a_{i j}} B_{i}(r)$

and the coefficients $\overline{a_{i j}}$ (which now replace $a_{i j k}$ in Eq. (3)) are determined by diagonalizing the $(N+1)$-electron Hamiltonian inside the R-matrix box that contained all bound atomic orbitals used for the description of C II levels. The relativistic effects in the scattering calculations have been incorporated in the BreitPauli Hamiltonian through the use of the Darwin, mass correction and spin-orbit operators. The radius of the R-matrix box was chosen to be $21.3 a_{0}$ and $86 \mathrm{~B}$-splines were used for the expansion of continuum orbitals. These parameters were sufficient to obtain converged results for a wide energy range up to about 12.5 Ryd. The B-spline R-matrix calculations were carried out for partial waves up to $J=37$. These partial waves were estimated normally to give converged cross sections for forbidden transitions. For the allowed transitions a top-up procedure based on the Coulomb-Bethe approximation was employed to estimate the contributions for $J$ larger than 37 . The top-up contributions for the non-dipole transitions have been estimated by assuming that the collision strengths form a geometric progression in $J$.

In many astrophysical applications it is convenient to use excitation rate coefficients or thermally averaged collision 
strengths as a function of electron temperature. The excitation rates are obtained by averaging collision strengths over a Maxwellian distribution of electron energies. The excitation rate coefficient for a transition from state $i$ to state $f$ at electron temperature $T_{\mathrm{e}}$ is given by

$C_{i f}=\frac{8.629 \times 10^{-6}}{g_{i} T_{\mathrm{e}}^{1 / 2}} \gamma_{i f}\left(T_{\mathrm{e}}\right) \exp \left(\frac{-\Delta E_{i f}}{k T_{\mathrm{e}}}\right) \mathrm{cm}^{3} \mathrm{~s}^{-1}$,

where $g_{i}$ is the statistical weight of the lower level $i, \Delta E_{i f}=E_{f}-$ $E_{i}$ is the excitation energy and $\gamma_{i f}$ is a dimensionless quantity called effective collision strength given by

$\gamma_{i f}\left(T_{\mathrm{e}}\right)=\int_{0}^{\infty} \Omega_{i f} \exp \left(\frac{-E_{f}}{k T_{\mathrm{e}}}\right) d\left(\frac{E_{f}}{k T_{\mathrm{e}}}\right)$,

where $E_{f}$ is the energy of incident electron with respect to the upper level $f$. If the collision strength is assumed to be independent of the incident electron energy, we have $\gamma_{i f}=\Omega_{i f}$. The effective collision strengths are calculated by integrating collision strengths for fine-structure levels over a Maxwellian distribution of electron energies. The integration in Eq. (6) should be carried out using energy dependent collision strengths from threshold to infinity. The collision strengths at higher energies are particularly important for the allowed transitions. The energy dependence of collision strengths for allowed transitions can be properly accounted by using extrapolation technique. In the asymptotic region, the collision strengths follow a high energy limiting behavior for the dipole-allowed transitions

$\Omega_{i f}(E) \sim_{E \rightarrow \infty} d \ln (E)$,

where the parameter $d$ is proportional to the oscillator strength. The collision strengths vary smoothly in the high energy region and exhibit an increasing trend for dipole-allowed transitions. The collision strength increases more rapidly for the stronger dipole-allowed transitions than the weaker transitions.

\section{Results and discussion}

In Table 1, we present a comparison of our calculated excitation energies with measured values from the NIST compilation (http://physics.nist.gov) and the atomic structure calculation of Correge \& Hibbert (2002). Correge \& Hibbert (2002) presented excitation energies with and without core correlation in the $1 \mathrm{~s}^{2}$ core and noted that the inclusion of core correlation improves slightly the agreement of calculated excitation energies with experiment. In Table 1 we have included their best results with core correlation. Our calculation does not include core correlation. The agreement between the calculated and measured excitation energies is normally better than $1 \%$. The energy ordering of the excitation levels also agree with experiment, except for the $2 \mathrm{~s} 2 \mathrm{p}^{2}{ }^{2} \mathrm{D}_{3 / 2,5 / 2}$ and $2 \mathrm{p}^{3}{ }^{2} \mathrm{P}_{3 / 2,5 / 2}$ levels. There is an excellent agreement between the present calculation and the calculation of Correge \& Hibbert (2002). The calculated results of Correge \& Hibbert (2002) are in slightly better agreement with experiment than the present calculation because of the inclusion of core correlation. A very good agreement with extensive structure calculation and experiment establishes a good quality of target wave functions used in our scattering calculation. To the best of our knowledge no earlier scattering calculation reported excitation energies for fine-structure levels. The energy splitting of the fine-structure levels in C II is very small indicating insignificance of relativistic effects.
Table 1. Excitation energies (Ry) of C II levels relative to the ground level. Present theory is compared with the NIST compilation (http:// physics . nist. gov) and Correge \& Hibbert (2002) (CH).

\begin{tabular}{|c|c|c|c|c|}
\hline$\overline{\text { Key }}$ & Level & Present & NIST & $\mathrm{CH}$ \\
\hline 1 & $2 s^{2} 2 p^{2} P_{1 / 2}$ & 0.00000 & 0.00000 & 0.00000 \\
\hline 2 & $2 \mathrm{~s}^{2} 2 \mathrm{p}^{2} \mathrm{P}_{3 / 2}$ & 0.00056 & 0.00058 & 0.00056 \\
\hline 3 & $2 \mathrm{~s} 2 \mathrm{p}^{2}{ }^{4} \mathrm{P}_{1 / 2}$ & 0.38942 & 0.39188 & 0.38930 \\
\hline 4 & $2 \mathrm{~s} 2 \mathrm{p}^{2}{ }^{4} \mathrm{P}_{3 / 2}$ & 0.38962 & 0.39208 & 0.38950 \\
\hline 5 & $2 \mathrm{~s} 2 \mathrm{p}^{2}{ }^{4} \mathrm{P}_{5 / 2}$ & 0.38995 & 0.39233 & 0.38975 \\
\hline 6 & $2 \mathrm{~s} 2 \mathrm{p}^{2}{ }^{2} \mathrm{D}_{3 / 2}$ & 0.69042 & 0.68284 & 0.68858 \\
\hline 7 & $2 \mathrm{~s} 2 \mathrm{p}^{2}{ }^{2} \mathrm{D}_{5 / 2}$ & 0.69045 & 0.68281 & 0.68856 \\
\hline 8 & $2 \mathrm{~s} 2 \mathrm{p}^{2}{ }^{2} \mathrm{~S}_{1 / 2}$ & 0.89105 & 0.87932 & 0.88750 \\
\hline 9 & $2 \mathrm{~s} 2 \mathrm{p}^{2}{ }^{2} \mathrm{P}_{1 / 2}$ & 1.02536 & 1.00808 & 1.01756 \\
\hline 10 & $2 \mathrm{~s} 2 \mathrm{p}^{2}{ }^{2} \mathrm{P}_{3 / 2}$ & 1.02577 & 1.00846 & 1.01793 \\
\hline 11 & $2 \mathrm{~s}^{2} 3 \mathrm{~s}^{2} \mathrm{~S}_{1 / 2}$ & 1.05822 & 1.06197 & 1.06178 \\
\hline 12 & $2 s^{2} 3 p^{2} P_{1 / 2}$ & 1.19362 & 1.20036 & 1.19692 \\
\hline 13 & $2 s^{2} 3 p^{2} P_{3 / 2}$ & 1.19372 & 1.20046 & 1.19702 \\
\hline 14 & $2 \mathrm{p}^{3}{ }^{3} S_{3 / 2}$ & 1.30139 & 1.29425 & 1.29279 \\
\hline 15 & $2 s^{2} 3 d^{2} D_{3 / 2}$ & 1.32894 & 1.32634 & 1.32497 \\
\hline 16 & $2 \mathrm{~s}^{2} 3 \mathrm{~d}^{2} \mathrm{D}_{5 / 2}$ & 1.32896 & 1.32636 & 1.32498 \\
\hline 17 & $2 \mathrm{p}^{3}{ }^{2} \mathrm{D}_{3 / 2}$ & 1.38707 & 1.37115 & 1.37994 \\
\hline 18 & $2 \mathrm{p}^{3}{ }^{2} \mathrm{D}_{5 / 2}$ & 1.38709 & 1.37111 & 1.37989 \\
\hline 19 & $2 s^{2} 4 s^{2} S_{1 / 2}$ & 1.43018 & 1.43282 & \\
\hline 20 & $2 s^{2} 4 p^{2} P_{1 / 2}$ & 1.49530 & 1.48097 & 1.47780 \\
\hline 21 & $2 s^{2} 4 p^{2} P_{3 / 2}$ & 1.49538 & 1.48103 & 1.48103 \\
\hline 22 & $2 \mathrm{~s} 2 \mathrm{p} 3 s{ }^{4} \mathrm{P}_{1 / 2}$ & 1.51711 & 1.52152 & \\
\hline 23 & $2 \mathrm{~s} 2 \mathrm{p} 3 s^{4} \mathrm{P}_{3 / 2}$ & 1.51736 & 1.52173 & \\
\hline 24 & $2 \mathrm{~s} 2 \mathrm{p} 3 s{ }^{4} \mathrm{P}_{5 / 2}$ & 1.51776 & 1.52214 & \\
\hline 25 & $2 \mathrm{~s}^{2} 4 \mathrm{~d}^{2} \mathrm{D}_{3 / 2}$ & 1.53172 & 1.53206 & \\
\hline 26 & $2 s^{2} 4 d^{2} D_{5 / 2}$ & 1.53174 & 1.53206 & \\
\hline 27 & $2 \mathrm{p}^{3}{ }^{2} \mathrm{P}_{1 / 2}$ & 1.55024 & 1.53758 & 1.54006 \\
\hline 28 & $2 \mathrm{p}^{3}{ }^{2} \mathrm{P}_{3 / 2}$ & 1.55048 & 1.53775 & 1.54025 \\
\hline 29 & $2 s^{2} 4 f^{2} F_{5 / 2}$ & 1.55788 & 1.53984 & \\
\hline 30 & $2 s^{2} 4 f^{2} F_{7 / 2}$ & 1.55789 & 1.53984 & \\
\hline 31 & $2 \mathrm{~s}^{2} 5 \mathrm{~s}^{2} \mathrm{~S}_{1 / 2}$ & 1.59212 & 1.57966 & \\
\hline 32 & $2 s^{2} 5 p^{2} P_{1 / 2}$ & 1.61817 & 1.59734 & 1.60208 \\
\hline 33 & $2 s^{2} 5 p^{2} \mathrm{P}_{3 / 2}$ & 1.61826 & 1.59740 & 1.60214 \\
\hline 34 & $2 \mathrm{~s} 2 \mathrm{p} 3 s^{2} \mathrm{P}_{1 / 2}$ & 1.63320 & 1.62000 & \\
\hline 35 & $2 \mathrm{~s} 2 \mathrm{p} 3 s^{2} \mathrm{P}_{3 / 2}$ & 1.63334 & 1.62017 & \\
\hline 36 & $2 \mathrm{~s}^{2} 5 \mathrm{~d}^{2} \mathrm{D}_{3 / 2}$ & 1.64584 & 1.62657 & \\
\hline 37 & $2 s^{2} 5 d^{2} D_{5 / 2}$ & 1.64586 & 1.62657 & \\
\hline 38 & $2 \mathrm{~s}^{2} 5 \mathrm{f}^{2} \mathrm{~F}_{5 / 2}$ & 1.64792 & 1.63077 & \\
\hline 39 & $2 s^{2} 5 f^{2} F_{7 / 2}$ & 1.64794 & 1.63077 & \\
\hline 40 & $2 s^{2} 6 s^{2} S_{1 / 2}$ & 1.66139 & 1.65180 & \\
\hline 41 & $2 \mathrm{~s} 2 \mathrm{p} 3 \mathrm{p}^{2} \mathrm{P}_{1 / 2}$ & 1.67228 & 1.65872 & \\
\hline 42 & $2 \mathrm{~s} 2 \mathrm{p} 3 \mathrm{p}^{2} \mathrm{P}_{3 / 2}$ & 1.67233 & 1.65890 & \\
\hline
\end{tabular}

The present oscillator strengths and transition probabilities for intercombination lines in the length form have been compared with the calculation of Tachiev \& Froese Fischer (2000) in Table 2. The values of oscillator strengths for intercombination lines are usually several orders of magnitude smaller than those for the allowed transitions. The intercombination lines are induced by the spin-orbit interaction by causing mixing between different LS symmetries with the same set of quantum numbers $J$ and $\pi$. There is reasonable agreement between the two calculations for the intercombination lines of the $2 s^{2} 2 p^{2} \mathrm{P}^{\circ}-$ $2 \mathrm{~s} 2 \mathrm{p}^{2}{ }^{4} \mathrm{P}$ multiplet. There is also a reasonable agreement between the two calculations for fine-structure components with significant strength. For example, oscillator strengths for the $2 \mathrm{~s} 2 \mathrm{p}^{2}{ }^{4} \mathrm{P}_{5 / 2}-2 \mathrm{~s}^{2} 3 \mathrm{p}{ }^{2} \mathrm{P}_{3 / 2}^{\circ}, 2 \mathrm{~s} 2 \mathrm{p}^{2}{ }^{4} \mathrm{P}_{5 / 2}-2 \mathrm{p}^{3}{ }^{2} \mathrm{D}_{5 / 2}, 2 \mathrm{~s} 2 \mathrm{p}^{2}{ }^{2} \mathrm{~S}_{1 / 2}-$ $2 \mathrm{~s} 2 \mathrm{p} 3 \mathrm{~s}{ }^{4} \mathrm{P}_{3 / 2}$ and $2 \mathrm{~s} 2 \mathrm{p}^{2}{ }^{2} \mathrm{P}_{3 / 2}-2 \mathrm{p}^{3}{ }^{4} \mathrm{~S}_{3 / 2}$ fine-structure transitions agree well. A comparison of present length transition probabilities for the dipole-allowed and intercombination transitions has 
been shown in Table 3 with other previous calculations. Our calculation agrees very well with the calculation of Tachiev \& Froese Fischer (2000) and Correge \& Hibbert (2002) for most fine-structure transitions. The CIV3 results from the calculation of Correge \& Hibbert (2002) include core correlation and are fine-tuned to experimental energies. Our results show some discrepancies with the calculation of Galavis et al. (1998) for several fine-structure transitions.

The length $\left(f_{L}\right)$ and velocity $\left(f_{V}\right)$ values of oscillator strengths and transition probabilities $\left(A_{L}\right.$ and $\left.A_{V}\right)$ for dipoleallowed transitions among fine-structure levels have been tabulated in Table 4, and our results have been compared with the calculation of Tachiev \& Froese Fischer (2000). We have reported results for the allowed transitions between the levels of doublet and quartet symmetries. The agreement between the length and velocity forms of oscillator strengths may to some extent indicate the accuracy of wave functions and the convergence of $\mathrm{CI}$ expansions. The convergence of results is an important accuracy criterion. There is normally a good agreement between the present length and velocity forms of oscillator strengths and with the results from the calculation of Tachiev \& Froese Fischer (2000). The agreement between our results and Tachiev $\&$ Froese Fischer (2000) is normally within $20 \%$ for most dipoleallowed transitions. The agreement provides us confidence in the accuracy of our target wave functions.

Accurate description of target wave functions is an essential part of a reliable scattering calculation. The quality of target wave functions used in our scattering calculation is very good as has been assessed by comparing computed excitation energies and oscillator strengths with experiment and other reliable calculations. We included 42 fine-structure levels arising from the $23 \mathrm{LS} 2 \mathrm{~s}^{2} 2 \mathrm{p}{ }^{2} \mathrm{P}^{\circ}, 2 \mathrm{~s} 2 \mathrm{p}^{2}{ }^{4} \mathrm{P},{ }^{2} \mathrm{D},{ }^{2} \mathrm{P},{ }^{2} \mathrm{~S}, 2 \mathrm{~s}^{2} n s(n=3-6){ }^{2} \mathrm{~S}$, $2 \mathrm{~s}^{2} n p(n=3-5){ }^{2} \mathrm{P}^{\circ}, 2 \mathrm{~s}^{2} n d(n=3-5){ }^{2} \mathrm{D}, 2 \mathrm{p}^{3}{ }^{4} S^{\circ}{ }^{2} \mathrm{P}^{\circ},{ }^{2} \mathrm{D}^{\circ}$, $2 \mathrm{~s}^{2} n f(n=4,5){ }^{2} \mathrm{~F}^{\circ}, 2 \mathrm{~s} 2 \mathrm{p} 3 \mathrm{~s}{ }^{4} \mathrm{P}^{\circ},{ }^{2} \mathrm{P}^{\circ}$ and $2 \mathrm{~s} 2 \mathrm{p} 3 \mathrm{p}{ }^{2} \mathrm{P}$ terms. We have plotted resonant collision strengths in the thresholds energy region and non-resonant background collision strengths above the highest excitation threshold energy region up to $2.0 \mathrm{Ryd}$ as a function of electron energy for the forbidden $2 \mathrm{~s}^{2} 2 \mathrm{p}^{2} \mathrm{P}_{1 / 2}^{\circ}$ ${ }^{2} \mathrm{P}_{3 / 2}^{\circ}$ and allowed $2 \mathrm{~s}^{2} 2 \mathrm{p}^{2} \mathrm{P}_{3 / 2}^{\circ}-2 \mathrm{~s} 2 \mathrm{p}^{2}{ }^{2} \mathrm{D}_{5 / 2}$ transitions in Figs. 1 and 2 respectively. It is clear from these figures that the resonance structures are complex and make significant enhancements in collision strengths. The non-resonant background collision strength for the allowed transition is larger than for the forbidden transition. The resonance enhancement in collision strengths for the forbidden transitions is normally larger compared to allowed transitions. The relativistic effects appear to be small for these transitions. We chose a fine energy mesh for collision strength calculation in the thresholds energy region to delineate the resonance structures. The collision problem in the external region was solved using an energy mesh of $0.0002 \mathrm{Ryd}$ in the closed-channels energy regions up to $1.658 \mathrm{Ryd}$. In the energy region of all open channels where there are no resonances and collision strengths show smooth variation we used an energy mesh of 0.2 Ryd. Resonance structures are quite dense in the energy region up to the $2 \mathrm{~s}^{2} 3 \mathrm{p}^{2} \mathrm{P}^{\circ}$ threshold around 1.20 Ryd. Our calculation properly includes important short-range correlation effect to ensure correct position of resonances in the low energy region.

A good agreement with the measured absolute direct excitation cross sections for the intercombination $2 s^{2} 2 p^{2} \mathrm{P}^{\circ}-2 \mathrm{~s} 2 \mathrm{p}^{2}{ }^{4} \mathrm{P}$ and resonance $2 \mathrm{~s}^{2} 2 \mathrm{p}^{2} \mathrm{P}^{\circ}-2 \mathrm{~s} 2 \mathrm{p}^{2}{ }^{2} \mathrm{D},{ }^{2} \mathrm{~S}$ transitions (Smith et al. 1996) has been obtained. The fine-structure components of these multiplets were not resolved in the experiment because for very

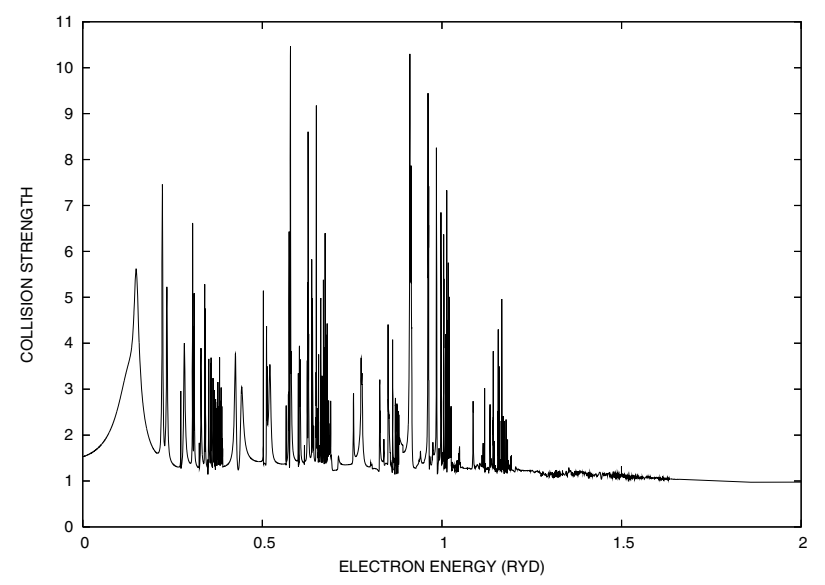

Fig. 1. Collision strength for the forbidden $2 \mathrm{~s}^{2} 2 \mathrm{p}^{2} \mathrm{P}_{1 / 2}^{\circ}{ }^{2} \mathrm{P}_{3 / 2}^{\circ}$ transition as a function of electron energy.

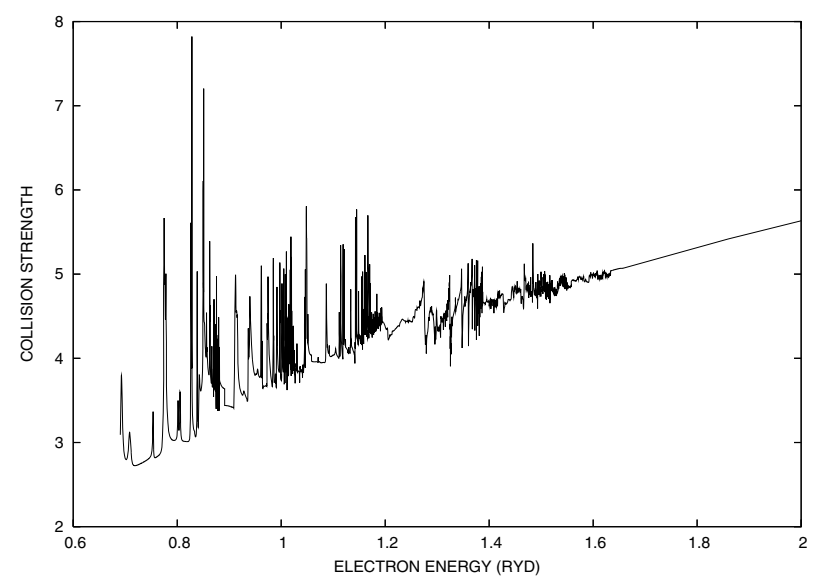

Fig. 2. Collision strength for the allowed $2 s^{2} 2 p^{2} P_{3 / 2}^{\circ}-2 s 2 p^{2}{ }^{2} D_{5 / 2}$ transition as a function of electron energy.

small separations between fine-structure levels. The uncertainty in experimental cross sections is about $20 \%$ and the energy resolution is $0.250 \mathrm{eV}$. We have convoluted theoretical cross sections to the experimental energy spread. The convoluted theoretical cross sections have been compared with the measured cross sections in Figs. 3-5. The convoluted theoretical cross sections for the intercombination transition in Fig. 3 are within experimental error bars for most incident electron energies except a few energies around $12 \mathrm{eV}$ and $15 \mathrm{eV}$ where experimental results are somewhat larger than the theory.

The excitation cross sections for the resonance $2 \mathrm{~s}^{2} 2 \mathrm{p}^{2} \mathrm{P}^{\circ}-$ $2 \mathrm{~s} 2 \mathrm{p}^{2}{ }^{2} \mathrm{D}$ and ${ }^{2} \mathrm{~S}$ transitions have been compared with measured cross sections in Figs. 4-5. Our theory shows good agreement with the measured cross sections for the ${ }^{2} \mathrm{P}^{\circ}-2 \mathrm{~s} 2 \mathrm{p}^{2}{ }^{2} \mathrm{D}$ transition in Fig. 4 at most incident electron energies. However, discrepancies exist at a few energies. Similar agreement between theory and experiment exists for the ${ }^{2} \mathrm{P}^{\circ}-2 \mathrm{~s} 2 \mathrm{p}^{2}{ }^{2} \mathrm{~S}$ transition shown in Fig. 5. The theoretical cross sections are within experimental uncertainty for all energies except two incident electron energies around $14 \mathrm{eV}$. The 8-state R-matrix theoretical cross sections from the work of Smith et al. (1996) have also been displayed in Figs. 3-5 by open squares. The two calculations normally agree to about $20 \%$, except for the ${ }^{2} \mathrm{P}^{\circ}-2 \mathrm{~s} 2 \mathrm{p}^{2}{ }^{2} \mathrm{~S}$ transition close to threshold energy where 8 -state calculation overestimates. The agreement between theory and experiment provides some additional indication that our collision strengths are likely to be 
Table 2. Oscillator strengths and transition probabilities for intercombination lines in C II. Present work is compared with the calculation of Tachiev \& Froese Fischer (2000) (TFF).

\begin{tabular}{|c|c|c|c|c|c|c|c|}
\hline \multirow[t]{2}{*}{ Transition } & \multirow[t]{2}{*}{$2 J$} & \multirow[t]{2}{*}{$2 J^{\prime}$} & \multirow[t]{2}{*}{$\lambda$} & \multicolumn{2}{|c|}{ Present results } & \multicolumn{2}{|c|}{ TFF } \\
\hline & & & & $f_{i k}$ & $A_{i k}$ & $f_{i k}$ & $A_{i k}$ \\
\hline \multirow[t]{5}{*}{$2 \mathrm{~s}^{2} 2 \mathrm{p}^{2} \mathrm{P}-2 \mathrm{~s} 2 \mathrm{p}^{2}{ }^{4} \mathrm{P}$} & 1 & 1 & 2322.45 & $4.27(-8)$ & $5.200(1)$ & $4.96(-8)$ & $6.136(1)$ \\
\hline & 1 & 3 & 2321.25 & $2.81(-9)$ & $1.711(0)$ & $2.36(-9)$ & $1.462(0)$ \\
\hline & 3 & 1 & 2324.89 & $2.51(-8)$ & $6.107(1)$ & $2.81(-8)$ & $6.929(1)$ \\
\hline & 3 & 3 & 2324.69 & $7.08(-9)$ & $8.606(0)$ & $7.17(-9)$ & $8.853(0)$ \\
\hline & 3 & 5 & 2323.15 & $4.24(-8)$ & $3.445(1)$ & $5.43(-8)$ & 4.477(1) \\
\hline \multirow[t]{5}{*}{$2 \mathrm{~s} 2 \mathrm{p}^{2}{ }^{4} \mathrm{P}-2 \mathrm{~s}^{2} 3 \mathrm{p}^{2} \mathrm{P}$} & 1 & 1 & 1125.98 & 7.64(-9) & $3.966(1)$ & $1.06(-8)$ & 5.564(1) \\
\hline & 1 & 3 & 1125.84 & $2.53(-8)$ & $6.582(1)$ & $3.85(-8)$ & $1.012(2)$ \\
\hline & 3 & 1 & 1126.26 & $1.75(-9)$ & $1.822(1)$ & $8.63(-10)$ & $9.080(0)$ \\
\hline & 3 & 3 & 1126.12 & $2.95(-9)$ & $1.532(1)$ & $3.65(-9)$ & $1.918(1)$ \\
\hline & 5 & 3 & 1126.48 & $2.19(-8)$ & $1.708(2)$ & $2.27(-8)$ & $1.786(2)$ \\
\hline \multirow[t]{5}{*}{$2 \mathrm{~s} 2 \mathrm{p}^{2}{ }^{4} \mathrm{P}-2 \mathrm{p}^{3}{ }^{2} \mathrm{D}$} & 1 & 3 & 928.98 & $5.09(-9)$ & $2.037(1)$ & $8.75(-10)$ & $3.380(0)$ \\
\hline & 3 & 3 & 929.17 & $4.61(-8)$ & $3.690(2)$ & $6.89(-8)$ & $5.326(2)$ \\
\hline & 3 & 5 & 929.22 & $2.88(-9)$ & $1.535(1)$ & $6.63(-9)$ & $3.413(1)$ \\
\hline & 5 & 3 & 929.42 & $1.28(-8)$ & $1.534(2)$ & $2.46(-9)$ & $2.850(1)$ \\
\hline & 5 & 5 & 929.46 & $1.89(-7)$ & $1.510(3)$ & $1.92(-7)$ & $1.482(3)$ \\
\hline \multirow[t]{2}{*}{$2 \mathrm{~s} 2 \mathrm{p}^{2}{ }^{2} \mathrm{D}-2 \mathrm{p}^{3}{ }^{4} \mathrm{~s}$} & 3 & 3 & 1490.01 & $3.07(-9)$ & $9.218(0)$ & $2.44(-9)$ & $7.340(0)$ \\
\hline & 5 & 3 & 1489.95 & $2.01(-8)$ & $9.047(1)$ & $4.48(-8)$ & $2.017(2)$ \\
\hline \multirow[t]{5}{*}{$2 \mathrm{~s} 2 \mathrm{p}^{2}{ }^{2} \mathrm{D}-2 \mathrm{~s} 2 \mathrm{p} 3 \mathrm{~s}{ }^{4} \mathrm{P}$} & 3 & 1 & 1087.53 & $7.12(-8)$ & $7.814(2)$ & $1.71(-6)$ & $1.926(4)$ \\
\hline & 3 & 3 & 1087.25 & $1.49(-6)$ & $8.394(3)$ & & \\
\hline & 3 & 5 & 1086.72 & $5.86(-9)$ & $2.147(1)$ & $9.77(-9)$ & $3.679(1)$ \\
\hline & 5 & 3 & 1087.22 & $1.99(-7)$ & $1.642(3)$ & $7.01(-6)$ & $5.929(4)$ \\
\hline & 5 & 5 & 1086.69 & $5.49(-8)$ & $3.016(2)$ & $1.41(-7)$ & $6.446(2)$ \\
\hline $2 s 2 p^{2}{ }^{2} S-2 p^{3}{ }^{4} S$ & 1 & 3 & 2197.60 & $3.34(-9)$ & $2.556(0)$ & $1.40(-8)$ & $9.681(0)$ \\
\hline \multirow[t]{2}{*}{$2 \mathrm{~s} 2 \mathrm{p}^{2}{ }^{2} \mathrm{~S}-2 \mathrm{~s} 2 \mathrm{p} 3 \mathrm{~s}{ }^{4} \mathrm{P}$} & 1 & 1 & 1421.63 & $5.29(-8)$ & $1.663(2)$ & $2.23(-8)$ & $7.346(1)$ \\
\hline & 1 & 3 & 1421.15 & $2.63(-7)$ & $4.146(2)$ & $1.80(-7)$ & $2.976(2)$ \\
\hline \multirow[t]{2}{*}{$2 \mathrm{~s} 2 \mathrm{p}^{2}{ }^{2} \mathrm{P}-2 \mathrm{p}^{3}{ }^{4} \mathrm{~S}$} & 1 & 3 & 3187.26 & $4.81(-8)$ & $1.472(1)$ & $7.30(-8)$ & $2.397(1)$ \\
\hline & 3 & 3 & 3191.50 & $1.18(-7)$ & $7.172(1)$ & $1.23(-7)$ & $8.065(1)$ \\
\hline
\end{tabular}

Table 3. Comparison of transition probabilities in C II with the calculations of Tachiev \& Froese Fischer (2000) (TFF), Correge \& Hibbert (2002) (CH), Froese Fischer (1994)(FF) and Galavis et al. (1998) (GMZ).

\begin{tabular}{cccccccc}
\hline \hline Transition & $2 J$ & $2 J^{\prime}$ & Present & TFF & CH & FF & GMZ \\
\hline $2 \mathrm{~s}^{2} 2 \mathrm{p}{ }^{2} \mathrm{P}-2 \mathrm{~s} 2 \mathrm{p}^{2}{ }^{4} \mathrm{P}$ & 1 & 1 & $5.200(1)$ & $6.136(1)$ & $5.916(1)$ & $6.21(1)$ & $7.191(1)$ \\
& 1 & 3 & $1.711(0)$ & $1.462(0)$ & $1.380(0)$ & $1.43(0)$ & $1.871(0)$ \\
& 3 & 1 & $6.107(1)$ & $6.929(1)$ & $6.710(1)$ & $6.89(1)$ & $8.189(1)$ \\
& 3 & 3 & $8.606(0)$ & $8.853(0)$ & $8.353(0)$ & $9.34(0)$ & $1.022(1)$ \\
$2 \mathrm{~s}^{2} 2 \mathrm{p}{ }^{2} \mathrm{P}-2 \mathrm{~s} 2 \mathrm{p}^{2}{ }^{2} \mathrm{~S}$ & 1 & 1 & $7.975(1)$ & $4.477(1)$ & $4.279(1)$ & $4.57(1)$ & $5.031(1)$ \\
& 3 & 1 & $1.575(9)$ & $7.434(8)$ & $7.467(8)$ & $6.21(8)$ & $7.191(8)$ \\
$2 \mathrm{~s}^{2} 2 \mathrm{p}{ }^{2} \mathrm{P}-2 \mathrm{~s} 2 \mathrm{p}^{2}{ }^{2} \mathrm{P}$ & 1 & 1 & $2.909(9)$ & $2.718(9)$ & $2.694(9)$ & $2.72(9)$ & $2.779(9)$ \\
& 3 & 1 & $1.467(9)$ & $1.371(9)$ & $1.359(9)$ & $1.37(9)$ & $1.402(9)$ \\
& 1 & 3 & $7.298(8)$ & $6.818(8)$ & $6.757(8)$ & $6.83(8)$ & $6.973(8)$ \\
$2 \mathrm{~s}^{2} 2 \mathrm{p}{ }^{2} \mathrm{P}-2 \mathrm{~s} 2 \mathrm{p}^{2}{ }^{2} \mathrm{D}$ & 3 & 3 & $3.648(9)$ & $3.408(9)$ & $3.378(9)$ & $3.42(9)$ & $3.485(9)$ \\
& 3 & 3 & $2.567(8)$ & $2.427(8)$ & $2.423(8)$ & $2.56(8)$ & $2.185(8)$ \\
$2 \mathrm{~s}^{2} 2 \mathrm{p}{ }^{2} \mathrm{P}-2 \mathrm{~s}^{2} 3 \mathrm{~s}{ }^{2} \mathrm{~S}$ & 3 & 5 & $5.080(7)$ & $4.796(7)$ & $4.791(7)$ & $5.07(7)$ & $4.315(7)$ \\
& 1 & 1 & $1.117(8)$ & $1.487(8)$ & $1.438(8)$ & & \\
$2 \mathrm{~s}^{2} 2 \mathrm{p}{ }^{2} \mathrm{P}-2 \mathrm{~s}^{2} 3 \mathrm{~d}{ }^{2} \mathrm{D}$ & 3 & 1 & $2.166(8)$ & $2.926(8)$ & $2.831(8)$ & & \\
& 3 & 3 & $2.322(9)$ & $2.353(9)$ & $2.297(9)$ & & \\
& 3 & 5 & $2.647(8)$ & $4.703(8)$ & $4.597(8)$ & & \\
\hline
\end{tabular}

accurate. In our B-spline R-matrix calculation the use of nonorthogonal orbitals considerably simplified the structure of the bound part of the close-coupling expansion and thus allowed a substantial reduction in undesirable pseudoresonances.

We have calculated thermally averaged collision strengths using Eq. (6) at temperatures in the range 1000-400000 K. In Table 5 we present effective collision strengths for all transitions between the lowest 37 fine-structure levels specified in Table 1. The keys of lower and upper levels of a transition are given in Table 1. The experimental wavelengths of transitions are also listed in Table 5. The effective collision strengths for the allowed transitions increase with increasing temperature. The effective collision strengths for the intercombination transitions decrease rapidly with increasing temperature in the high temperature regime. These transitions can only occur through electron exchange. The strength of the spin-orbit interaction appears to be insignificant particularly for low-lying excited levels.

Our effective collision strengths for the forbidden $2 \mathrm{~s}^{2} 2 \mathrm{p}^{2} \mathrm{P}_{1 / 2}^{\circ}-{ }^{2} \mathrm{P}_{3 / 2}^{\circ}$ transition calculated in 42-level and 35-level calculations have been compared with the 10-state and 16-state 


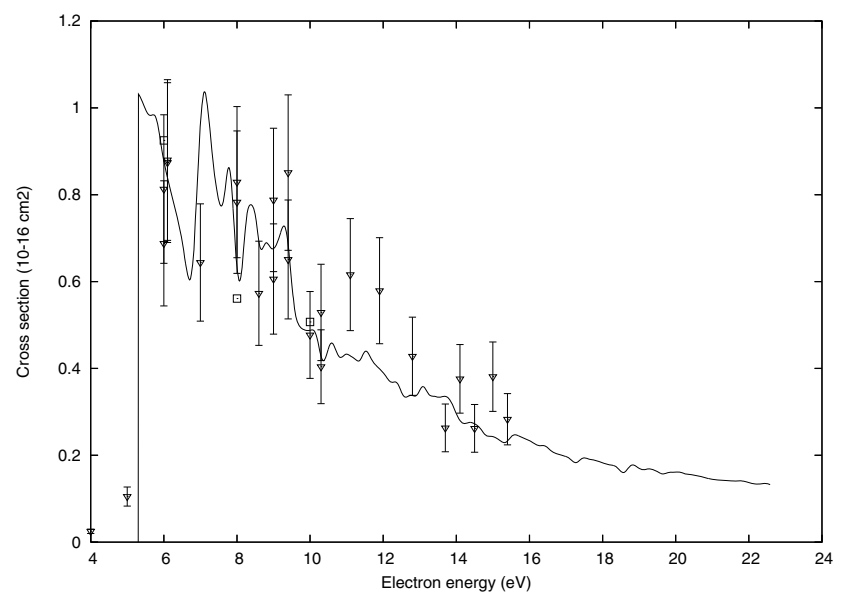

Fig. 3. Excitation cross sections for the $2 s^{2} 2 p^{2} \mathrm{P}^{\circ}-2 s 2 p^{2}{ }^{4} \mathrm{P}$ transition as a function of electron energy. Solid curve, present results; open squares, 8-state R-matrix results (Smith et al. 1996); open inverted triangles, experimental results of Smith et al. (1996); horizontal bar, measured results of Lafyatis \& Kohl (1987).

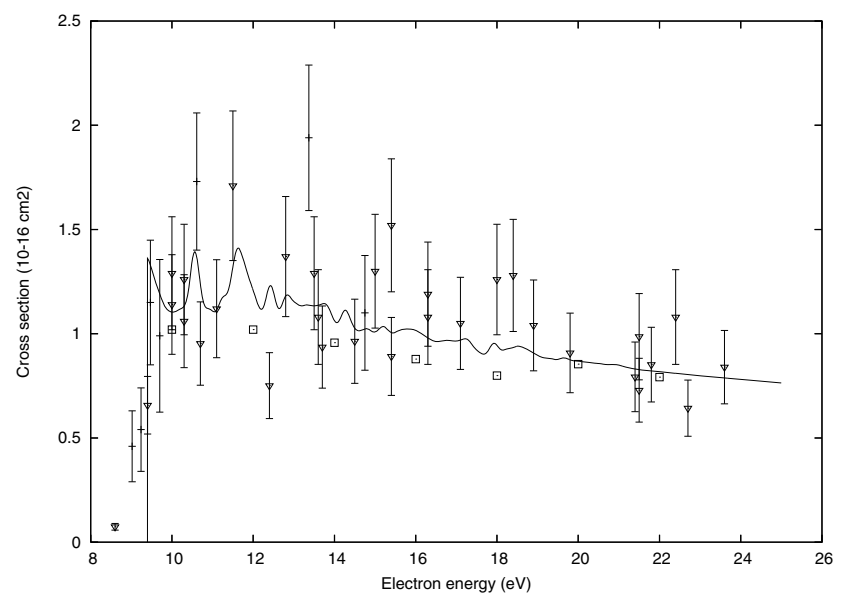

Fig. 4. Excitation cross sections for the $2 \mathrm{~s}^{2} 2 \mathrm{p}^{2} \mathrm{P}^{\circ}-2 \mathrm{~s} 2 \mathrm{p}^{2}{ }^{2} \mathrm{D}$ transition as a function of electron energy. Solid curve, present results; open squares, 8-state R-matrix results Smith et al. (1996); horizontal bars, measured results of Lafyatis \& Kohl (1987); open inverted triangles, experimental results of (Smith et al. 1996).

R-matrix calculations of Blum \& Pradhan (1992) and Wilson et al. (2005) in Fig. 6. The present results have been shown by solid curve (42-level) and short-dashed curve (35-level) and those of Blum \& Pradhan (1992) and Wilson et al. (2005) are displayed by dotted and long-dashed curves respectively in the temperature region from $\log T=2.5$ to $6.0 \mathrm{~K}$. Blum \& Pradhan (1992) reported effective collision strengths in the temperature range from $1000 \mathrm{~K}$ to $40000 \mathrm{~K}$ and Wilson et al. (2005) presented effective collision strengths for temperatures in the range $\log T=3.00-5.5 \mathrm{~K}$. The results from the previous calculations of Hayes \& Nussbaumer (1984) and Lennon et al. (1985) are also shown. Hayes \& Nussbaumer (1984) reported two sets of effective collision strengths with main resonances at calculated position and with main resonances shifted to experimental position. We have displayed both results in Fig. 6 by short-dash-dotted with calculated position and by long dash-dotted curve with resonances shifted to the experimental position. The 42-level and 35-level results are almost inseparable, indicating convergence of collision strengths. The various

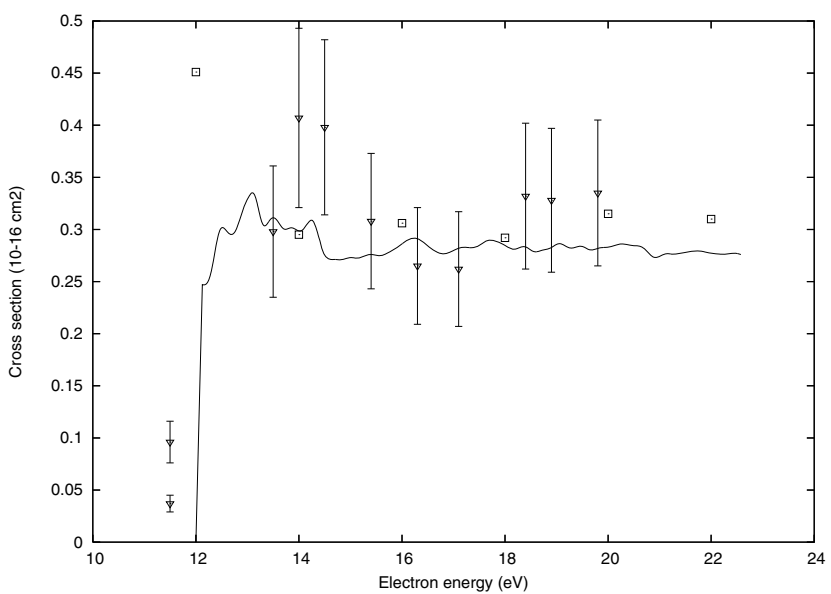

Fig. 5. Excitation cross sections for the $2 \mathrm{~s}^{2} 2 \mathrm{p}^{2} \mathrm{P}^{\circ}-2 \mathrm{~s} 2 \mathrm{p}^{2}{ }^{2} \mathrm{~S}$ transition as a function of electron energy. Solid curve, present results; open squares, 8-state R-matrix results (Smith et al. 1996); open inverted triangles, experimental results (Smith et al. 1996).

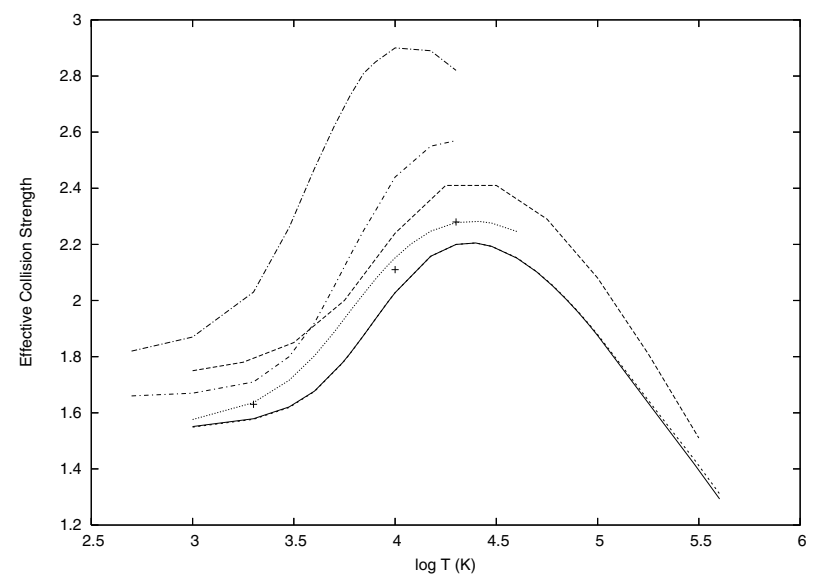

Fig. 6. Effective collision strengths for the $2 s^{2} 2 p{ }^{2} \mathrm{P}_{1 / 2}^{\circ}-2 s^{2} 2 p{ }^{2} \mathrm{P}_{3 / 2}^{\circ}$ transition as a function of electron temperature. Solid curve, present 42-level results; short-dashed curve, present 35-level results; longdashed curve, 16-state R-matrix results (Wilson et al. 2005); dotted curve, 10-state R-matrix results (Blum \& Pradhan 1992); long-dash dotted curve, calculation of Hayes \& Nussbaumer (1984) [experimental resonance positions]; short-dash dotted curve, calculation of Hayes \& Nussbaumer (1984) [calculated resonance positions]; pluses, calculation of Lennon et al. (1985).

calculations except that of Hayes \& Nussbaumer (1984) are normally within $10-15 \%$ of the present results. Our results are lower than the other calculations. The effective collision strengths for the intercombination $2 \mathrm{~s}^{2} 2 \mathrm{p}^{2} \mathrm{P}_{1 / 2}^{\circ}-2 \mathrm{~s} 2 \mathrm{p}^{2}{ }^{4} \mathrm{P}_{3 / 2}$ and $2 s 2 p^{2}{ }^{4} P_{1 / 2}-2 s 2 p^{2}{ }^{2} D_{5 / 2}$ transitions have been displayed in Figs. 7 and 8 respectively where our results (42-level: solid curve; 35-level: short dashed curve) have been compared with 10-state and 16-state results together with the results from Hayes \& Nussbaumer (1984) and Lennon et al. (1985). Our results show some qualitative and quantitative differences with previous calculations. The differences may have been caused by the combined differences in resonance structures and in background collision strengths which in turn may have been caused by the inaccuracies in target wave functions in previous calculations. Our results are about $13 \%$ smaller than the calculation of Wilson et al. (2005) in the lower temperature region, but the two calculations show excellent agreement at 


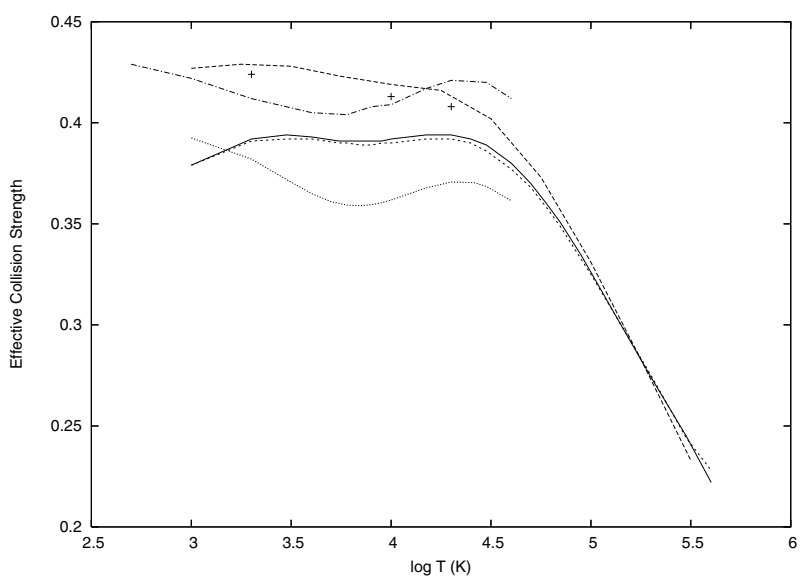

Fig. 7. Effective collision strengths for the $2 s^{2} 2 p{ }^{2} \mathrm{P}_{3 / 2}^{\circ}-2 s 2 \mathrm{p}^{2}{ }^{4} \mathrm{P}_{3 / 2}$ transition as a function of electron temperature. Solid curve, present 42-level results; short-dashed curve, present 35-level results; longdashed curve, 16-state R-matrix results (Wilson et al. 2005); dotted curve, 10-state R-matrix results (Blum \& Pradhan 1992); dash-dotted curve, calculation of Hayes \& Nussbaumer (1984); pluses, calculation of Lennon et al. (1985).

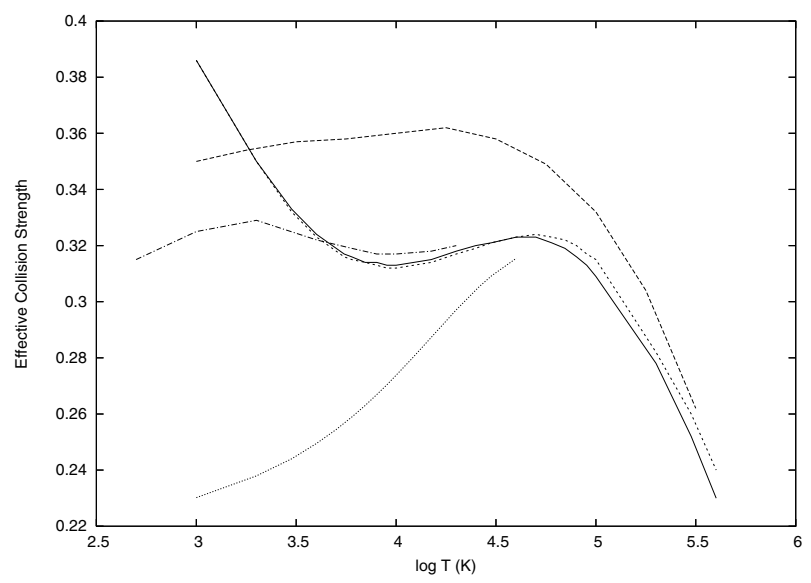

Fig. 8. Effective collision strengths for the $2 \mathrm{~s} 2 \mathrm{p}^{2}{ }^{4} \mathrm{P}_{1 / 2}-2 \mathrm{~s} 2 \mathrm{p}^{2}{ }^{2} \mathrm{D}_{5 / 2}$ transition as a function of electron temperature. Solid curve, present 42-level results; short-dashed curve, present 35-level results; longdashed curve, 16-state R-matrix results (Wilson et al. 2005); dotted curve, 10-state R-matrix results (Blum \& Pradhan 1992); long-dash dotted curve, calculation of Hayes \& Nussbaumer (1984).

higher temperatures for the ${ }^{2} \mathrm{P}_{1 / 2}^{\circ}-2 \mathrm{~s} 2 \mathrm{p}^{2}{ }^{4} \mathrm{P}_{3 / 2}$ transition. Larger discrepancies in shape and magnitude of effective collision strengths from various calculations exist, particularly at lower temperatures for the $2 s 2 p^{2}{ }^{4} \mathrm{P}_{1 / 2}-2 s 2 \mathrm{p}^{2}{ }^{2} \mathrm{D}_{5 / 2}$ transition. Our results differ by almost a factor of two with the calculation of Blum \& Pradhan (1992) at lower temperatures. Again there is a very good agreement with the calculation of Wilson et al. (2005) at higher temperatures.

Our results for the allowed $2 \mathrm{~s} 2 \mathrm{p}^{2}{ }^{2} \mathrm{D}_{3 / 2}-2 \mathrm{p}^{3}{ }^{2} \mathrm{D}_{5 / 2}^{\circ}$ and ${ }^{2} \mathrm{P}_{3 / 2}^{\circ}$ transitions are shown in Figs. 9 and 10. The results from the 10 -state and 16-state R-matrix calculations are also included. Our results show significant differences with the previous calculations. Our results differ by up to $40 \%$ with the calculation of Wilson et al. (2005) at lower temperatures for the $2 \mathrm{~s} 2 \mathrm{p}^{2}{ }^{2} \mathrm{D}_{3 / 2}-$ $2 \mathrm{p}^{3}{ }^{2} \mathrm{D}_{3 / 2}^{\circ}$ transition. Although there is an agreement in the shape between the present effective collision strengths and Blum \& Pradhan (1992) for the $2 \mathrm{~s} 2 \mathrm{p}^{2}{ }^{2} \mathrm{D}_{3 / 2}-2 \mathrm{p}^{3}{ }^{2} \mathrm{P}_{3 / 2}^{\circ}$ transition, there are substantial differences in magnitude. The calculation of

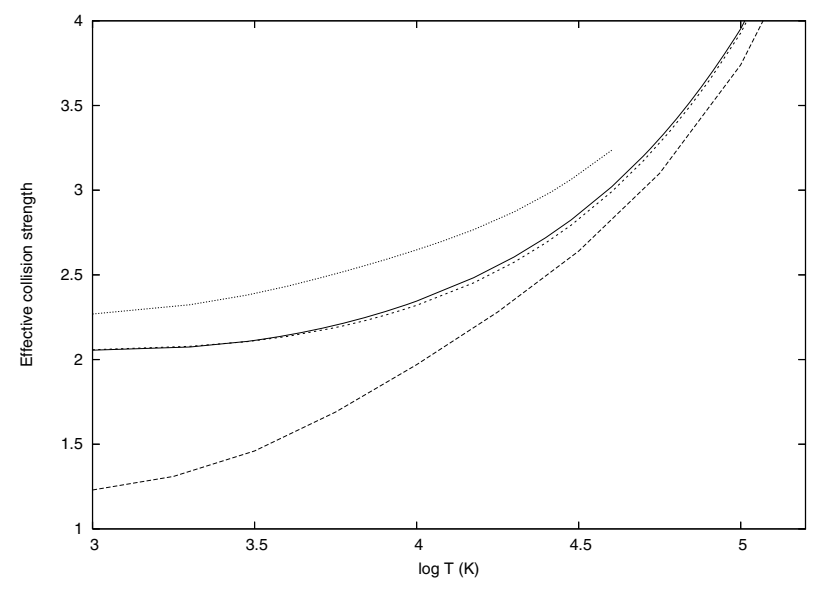

Fig. 9. Effective collision strengths for the $2 s 2 p^{2}{ }^{2} D_{3 / 2}-2 p^{3}{ }^{2} D_{5 / 2}^{\circ}$ transition as a function of electron temperature. Solid curve, present 42 -level results; dashed curve, present 35-level results; long-dashed curve, 16-state R-matrix results (Wilson et al. 2005); dotted curve, 10-state R-matrix results (Blum \& Pradhan 1992).

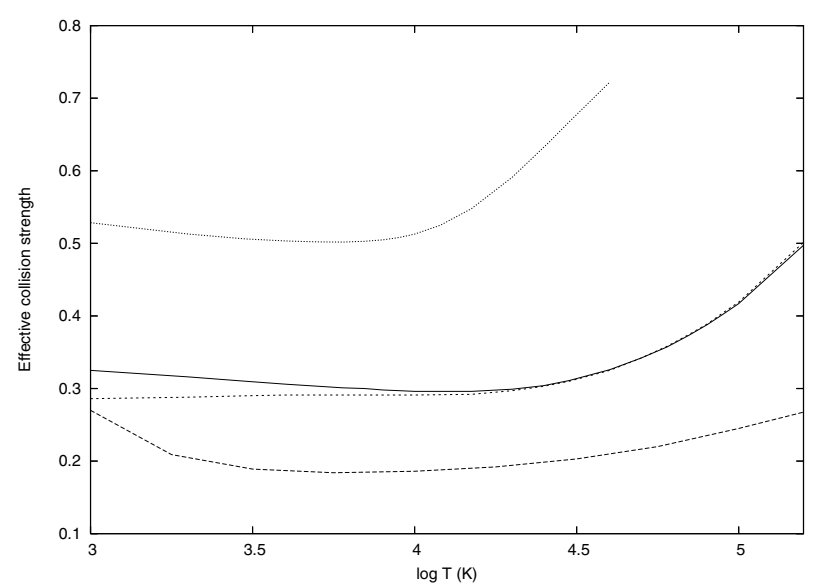

Fig. 10. Effective collision strengths for the $2 s 2 p^{2}{ }^{2} D_{3 / 2}-2 p^{3} \quad{ }^{2} P_{3 / 2}^{\circ}$ transition as a function of electron temperature. Solid curve, present 42-level results; short-dashed curve, present 35-level results; longdashed curve, 16-state R-matrix results (Wilson et al. 2005); dotted curve, 10-state R-matrix results (Blum \& Pradhan 1992).

Blum \& Pradhan (1992) is about $80 \%$ larger than the present calculation. It is clear from Figs. 6-10 that the present 42-level and 35-level calculations agree very well with each other indicating a good convergence of results. We carried out ab initio Rmatrix calculations for $J=0-37$ while the previous 10 -state and 16-state calculations considered $L=0-12$ partial waves which may also cause a part of discrepancies with these two calculations. Based upon the accurate description of target wave functions and scattering model, our results are likely to be more accurate than the previous calculations.

In conclusion, we have presented accurate oscillator strengths and effective collision strengths for C II lines among the fine-structure levels. We used non-orthogonal orbitals for the construction of CI wave functions for target levels and for the representation of scattering functions. The checks on excitation energies and oscillator strengths provide us confidence that our target wave functions are likely to be accurate to yield reliable collision strengths from the Breit-Pauli B-spline R-matrix scattering calculations. The use of non-orthogonal orbitals considerably simplified the structure of the bound part of the 
close-coupling expansion. We have attempted to account for important physical effects such as electron correlation, relativistic, and channel coupling effects by including coupling to higher excited target states. We believe our results to be most extensive and definitive to date. The Rydberg series of resonances converging to several excited levels are included in our calculation. The effective collision strengths are presented over a wide range of electron temperatures suitable for use in astrophysical plasmas modeling. These data are of considerable interest in astrophysical applications.

Acknowledgements. This research work is supported by NASA grant NNG06GD39G from Astronomy and Physics Research Analysis program.

\section{References}

Blum, R. D., \& Pradhan, A. K. 1991, Phys. Rev. A, 44, 6123 Blum, R. D., \& Pradhan, A. K. 1992, ApJS, 80, 425

Brage, T., Froese Fischer, C., \& Judge, P. G. 1995, ApJ, 445, 457 Correge, G., \& Hibbert, A. 2002, J. Phys. B, 35, 1211
Eissner, W., J. M., \& Nussbaumer, H. 1974, Comput. Phys. Commun., 8, 270 Froese Fischer, C. 1991, Comput. Phys. Commun., 64, 369

Froese Fischer, C. 1994, Phys. Scr., 49, 323

Galavis, M. E., Mendoza, C., \& Zeippen, C. J. 1998, A\&A, 131, 499

Hayes, M. A., \& Nussbaumer, H. 1984, A\&A, 134, 193

Hibbert, A. 1975, Comput. Phys. Commun., 9, 141

Keenan, F. P., Lennon, D. J., Johnson, C. T., \& Kingston, A. 1986, MNRAS, 220, 571

Lafyatis, G. P., \& Kohl, J. L. 1987, Phys. Rev. A, 36, 59

Lennon, D. J., Dufton, P. L., Hibbert, A., \& Kingston, A. E. 1985, ApJ, 294, 200 Luo, D., \& Pradhan, A. K. 1990, Phys. Rev. A, 41, 165

Nussbaumer, H., \& Storey, P. J. 1981, A\&A, 96, 91

Smith, S. J., Zuo, M., Chutjian, A., Tayal, S. S., \& Williams, I. D. 1996, ApJ, 440, 421

Tachiev, G. I., \& Froese Fischer, C. 2000, J. Phys. B, 33, 2419

Walborn, N. R., et al. 2002, ApJS, 154, 651

Williams, I. D., Greenwood, J. B., Srigengan, B., et al. 1998, Mass. Sci. Technol., 9, 930

Wilson, N. J., \& Bell, K. L. 2002, MNRAS, 337, 1027

Wilson, N. J., Bell, K. L., \& Hudson, C. E. 2005, A\&A, 432, 731

Zatsarinny, O. 2006, Comput. Phys. Commun., 124, 247

Zatsarinny, O., \& Bartschat, K. 2004, J. Phys. B, 37, 2173

Zatsarinny, O., \& Froese Fischer, C. 2000, Comput. Phys. Commun., 124, 247

Zatsarinny, O., \& Tayal, S. S. 2001, J. Phys. B, 34, 1299 